\title{
Analysis of selected pro- and anti-inflammatory cytokines in patients with multiple injuries in the early period after trauma
}

\author{
KATARZYNA GOŁĄBEK-DROPIEWSKA ${ }^{1}$, JUSTYNA PAWŁOWSKA', JACEK WITKOWSKI ${ }^{2}$, \\ JERZY LASEK ${ }^{3}$, WOJCIECH MARKS ${ }^{3}$, MARIUSZ STASIAK ${ }^{1}$, DAWID JASKÓLSKI ${ }^{1}$, \\ ALEKSANDRA KAWECKA ${ }^{l}$, PIOTR ŁUCZKIEWICZ ${ }^{1}$, BOGUSŁAW BACZKOWSKI \\ ${ }^{1} 2^{\text {nd }}$ Department of Orthopaedics and Kinetic Organ Traumatology, Medical University of Gdańsk, Poland \\ ${ }^{2}$ Chair and Department of Physiopathology, Medical University of Gdańsk, Poland \\ ${ }^{3}$ Department of Surgical and Multiorgan Trauma Training, Medical University of Gdańsk, Poland
}

\begin{abstract}
Introduction: Severe trauma causes damage to the protective barriers of the organism, and thus activates immunological reaction. Among substances secreted during this process pro-inflammatory cytokines are of high importance.

The aim of the study: Severe trauma causing multiple injuries is more likely to lead to particularly intensive inflammatory reaction, which can sometimes lead to serious complications, even life-threatening. The aim of the study is to determine those parameters which may serve as predictors of infectious complications and to enable estimation of the patient's immunological status before the decision to introduce elective procedures.

Material and methods: The study population included patients with multiple trauma treated in the Department of Trauma Surgery of the Medical University of Gdansk. The severity of injuries was evaluated with commonly used numerical scales (Revised Trauma Score - RTS, Injury Severity Score - ISS, Glasgow Coma Scale-GCS). Blood samples were collected on the first, second, and fifth day after injury. Evaluated parameters: C-reactive protein $(C R P)$, the level of cytokines: $I L-8, I L-1 \beta, I L-6, T N F$, $I L-12 p 70$, and IL-10. Control population: individuals without injury.

Results: Evaluation of IL-6, IL-8, and CRP levels in patients with multiple trauma in the early period after injury (2-3 days) could be considered as a predictor of delayed infection (5-10 days). CRP level, being cheap and commonly accessible, can be used in clinical practice enabling identification of patients at higher risk of infectious complications and introduction of appropriate treatment and prevention. The analysis of the mentioned parameters may contribute to choosing an appropriate management strategy, including "timing" depending on the patient's biological status.
\end{abstract}

Key words: cytokines, inflammation, $C R P$, infectious complications, multiple injuries.

(Centr Eur J Immunol 2018; 43 (1): 42-49)

\section{Introduction}

Every trauma leads to damage of the organism's protective barriers, and consequently provokes an inflammatory reaction. Destroyed cells secrete substances activating and attracting neutrophils, B and T lymphocytes, platelets, coagulation factors, and fibroblasts. Cytokines are the most important factors inducing and regulating this process. That complex reaction is supposed to reinstate homeostasis in the organism, limit the damage, and avoid infection. Nevertheless, any disturbances leading to overstimulation or suppression of the inflammatory response can cause severe complications, sometimes even life-threatening [1-7].

In this study, multiple injuries (polytrauma) were defined as injuries concerning at least two anatomical areas, each of them individually requiring hospitalisation or specialised treatment. This definition also covers other descriptions concerning Injury Severity Score (ISS) levels over 17 or 18 [8]. In those patients, inflammatory reaction concerns not only the area of injuries but is also usually generalised [9]. Such intensive response may lead to systemic inflammatory response syndrome

Correspondence: Katarzyna Gołąbek-Dropiewska, PhD, $2^{\text {nd }}$ Clinic of Orthopaedics \& Kinetic Organ Traumatology, Medical University of Gdańsk, Smoluchowskiego 17, 80-214 Gdańsk, Poland, e-mail: ottoll@o2.pl

Submitted: 29.06.2016; Accepted: 25.09.2016 
Table 1. Clinical characterisation of the studied patients

\begin{tabular}{lccccccc}
\hline $\begin{array}{l}\text { Features } \\
\text { \% of patients }\end{array}$ & $\begin{array}{c}\text { Head and neck } \\
\text { injury }\end{array}$ & Chest injury & $\begin{array}{c}\text { Abdomen and/ } \\
\text { or pelvis injury }\end{array}$ & $\begin{array}{c}\text { Extremities } \\
\text { injury }\end{array}$ & $\begin{array}{c}\text { Delayed surgical } \\
\text { procedure }\end{array}$ & $\begin{array}{c}\text { Infectious } \\
\text { complications }\end{array}$ & Sepsis \\
\hline Yes & $76 \%$ & $68 \%$ & $36 \%$ & $74 \%$ & $48 \%$ & $32 \%$ & $6 \%$ \\
\hline No & $24 \%$ & $32 \%$ & $64 \%$ & $26 \%$ & $52 \%$ & $68 \%$ & $94 \%$ \\
\hline
\end{tabular}

(SIRS), which potentially causes multiple organ dysfunction syndrome (MODS) and multiple organ failure (MOF), whereas excessive anti-inflammatory response in patients with polytrauma may result in compensatory antiinflammatory response syndrome (CARS) predisposing to infective complications [1-5, 12, 13].

The secretion of pro- and anti-inflammatory cytokines changes in particular phases of reaction modulating this process by activation or suppression of inflammatory cells. Any instability of cytokines level may lead to the aforementioned syndromes that threaten the patient's homeostasis and impede the therapy [1, 2, 4-7, 12]. Assessment of the changes of levels of cytokines early after injury may be helpful in predicting threats and preventing them.

\section{Material and methods}

Fifty patients with multiple injuries treated in the Department of Trauma Surgery of the Medical University of Gdańsk were included in the study. The population counted 50 patients -18 female and 32 male $(36 / 64 \%)$. The average age was 39.5 years, the youngest patient was 18 years old, and the oldest 92 years.

Exclusion criteria were:

- known autoimmune diseases or immunodeficiency,

- chronic receiving of corticosteroids or other immunosuppressive drugs,

- five years of cancer treatment history.

Patient consent in written form was obtained before taking the first blood sample. The Independent Commission of Bioethics approved this research - decision number: NKEBN/37/2008. Blood samples were collected on the first, second, and fifth day after trauma. The following cytokines were evaluated: IL-8, IL-1 $\beta$, IL-6, TNF, IL12p70, and IL-10. Additionally, C-reactive protein (CRP) levels were measured consecutively.

The control group included healthy volunteers who gave their written consent to participate in the study. The population counted 20 patients -8 female and 12 male (40/60\%). The average age was 40 years, the youngest participant was 23 years old, and the oldest 76 years.

The same exclusion criteria were used as in the test group. Blood samples were collected from volunteers once.

Commonly used numerical severity scales (ISS, GCS - Glasgow Coma Scale, RTS - Revised Trauma Score) were utilised in the assessment of the severity of the injury.
Cytokines levels were measured by fluorescence flow cytometry using fluorescently labelled antibodies. Clotted blood, taken on the day of collection, was centrifuged, and the supernatant was harvested and frozen at $-18^{\circ} \mathrm{C}$. After collecting all the samples the test was performed using a CBA Human Inflammatory Cytokines Kit from BD Biosciences. This method uses immunological reaction "antigenantibody" to identify particular substances in blood serum. Components of different fluorescence intensities are coated with the corresponding antibodies for individual cytokines - IL-8, IL-1 $\beta$, IL-6, IL-10, TNF, and IL-12p70. After preparing and incubating the mixture of tested serum with fluorescent substances, an immunological reaction occurred, leading to the connection of individual cytokine molecules with antibodies on fluorescent substance beads, forming the so-called sandwich complexes with different intensities of fluorescence. The test was performed using a flow cytometer (FACScan Becton and Dickinson, USA) in the Department of Physiopathology of the Medical University of Gdańsk.

Statistical analyses were performed with the Statsoft Statistica data analysis software system version 8 . $P$-values $\leq 0.05$ were taken to be statistical significance in applied tests. $U$-The Mann-Whitney test was performed to evaluate differences between patients and control compared groups, and the Wilcoxon test was applied to calculate differences in cytokine values between days. $P$ values less than 0.05 were considered as statistically significant, and above 0.05 and under 0.10 as borderline statistical significance. For analyses of correlation Pearsons correlation test was used. For specificity and sensitivity evaluation, ROC analysis was applied.

\section{Results}

The injured anatomical area of the body, delayed surgical procedures, and the presence of infectious complication (wound infection, pneumonia, sepsis) were evaluated (Table 1).

Table 2. Severity of injuries

\begin{tabular}{lcc}
\hline Values & $\begin{array}{c}\text { Injury Severity } \\
\text { Score (ISS) }\end{array}$ & $\begin{array}{c}\text { Glasgow Coma Scale (GCS) } \\
\text { at the time of admission to } \\
\text { emergency room }\end{array}$ \\
\hline Minimum & 18 & 9 \\
\hline Maximum & 41 & 15 \\
\hline Median & 26 & 14 \\
\hline
\end{tabular}


Table 3. IL-6 and IL-8 levels on the first, second, and fifth day after trauma and in controls

\begin{tabular}{lcccccccc}
\hline Values & \multicolumn{1}{c}{ IL-6 $(\mathbf{p g} / \mathbf{m l})$} & \multicolumn{3}{c}{ IL-8 (pg/ml) } \\
\hline Day/controls & I & II & V & Controls & I & II & V & Controls \\
\hline Minimum & 8.6 & 1.8 & 1.4 & 0.6 & 11.3 & 8.1 & 8.1 & 2.2 \\
\hline Maximum & 189.4 & 295.9 & 295.8 & 2.7 & 98.5 & 108 & 108 & 10.8 \\
\hline Median & 10.65 & 10 & 13.6 & 1.75 & 11.4 & 16.4 & 16.75 & 5.3 \\
\hline
\end{tabular}

Table 4. C-reactive protein (CRP) levels on particular days after injury in patients with multiple injuries $(\mathrm{Gb}) \mathrm{mg} / \mathrm{l}$

\begin{tabular}{lccc}
\hline CRP (mg/l) & $\begin{array}{c}\text { Day I } \\
\text { after injury }\end{array}$ & $\begin{array}{c}\text { Day II } \\
\text { after injury }\end{array}$ & $\begin{array}{c}\text { Day V } \\
\text { after injury }\end{array}$ \\
\hline Minimum & 0.4 & 7.46 & 1.86 \\
\hline Maximum & 202.1 & 278.8 & 315.5 \\
\hline Median & 36.5 & 68.2 & 59.15 \\
\hline
\end{tabular}
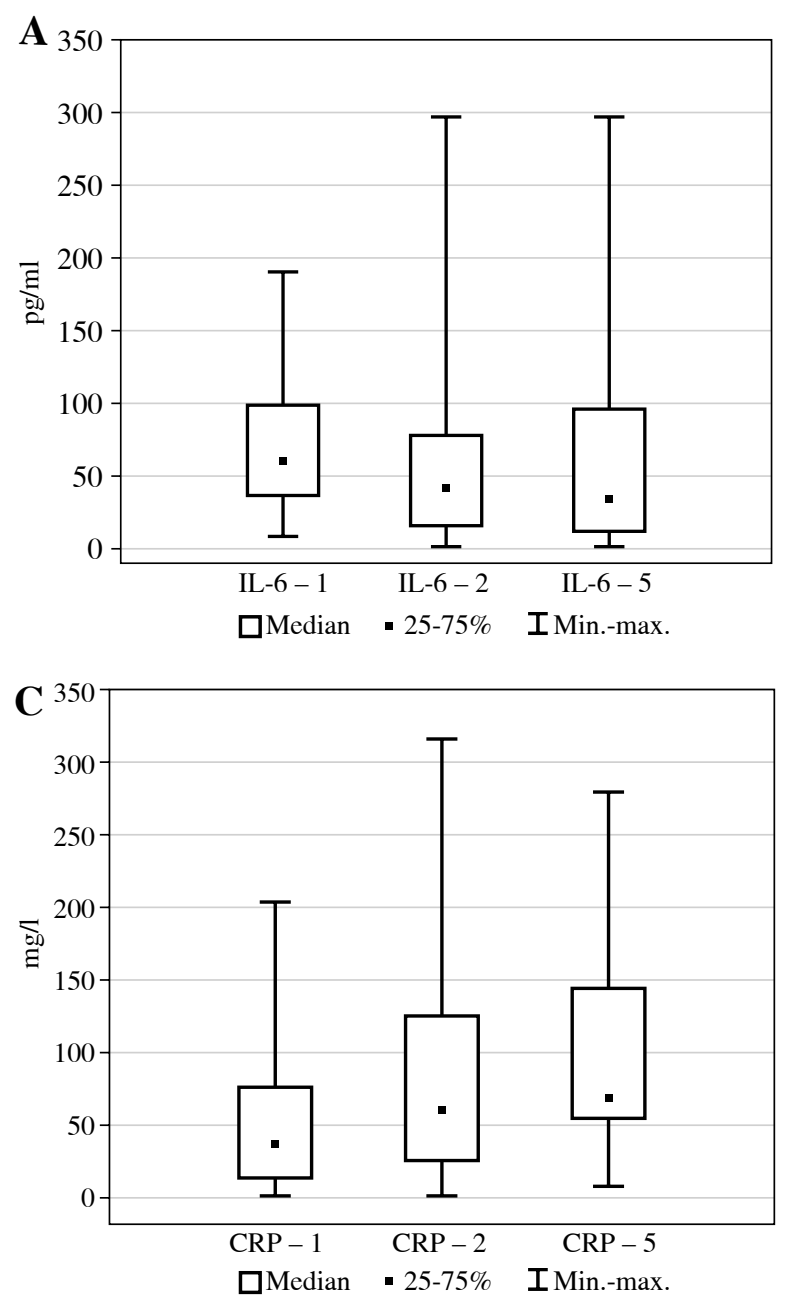

Delayed surgical procedures were introduced between the second and fifteenth day after trauma; the median was the fourth day. ISS and GCS were used in the assessment of the severity of injuries in the study group (Table 2).

Among tested cytokines there was no increase in levels of IL-1 $\beta$, TNF, and IL-12p70 compared to healthy subjects. These values floated around zero. Only in four patients was an increase in IL-10 level observed, but it concerned only particular measurements. Significant changes were found in IL-6 and IL-8 levels (Table 3).

CRP levels on particular days after injury were additionally evaluated (Table 4).

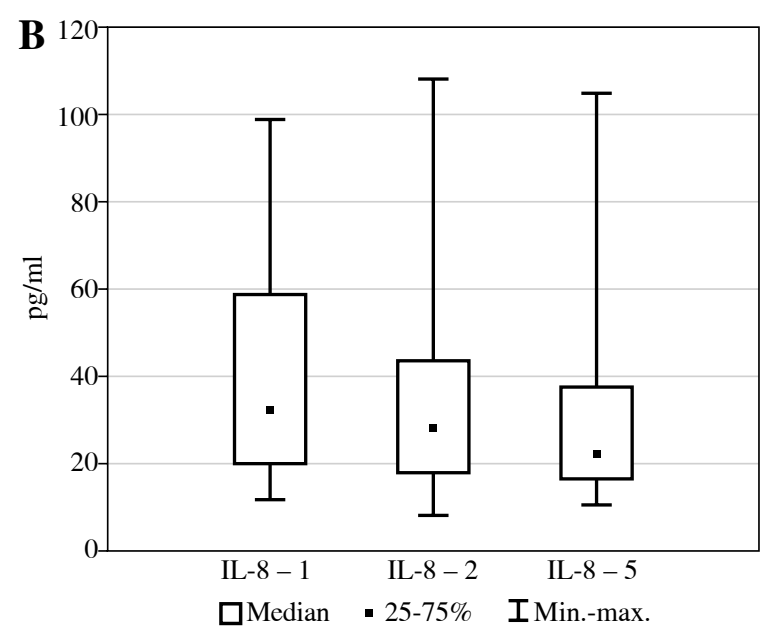

Fig. 1. Box-and-whisker plots present the differences between A) IL-6 level on the first, second, and fifth day after injury, B) IL-8 level on the first, second, and fifth day after injury, C) CRP levels in particular days after injury 
The IL-6 level was the highest on the first day after injury and then decreased on the second and the fifth day. The differences are on the border of statistical significance. IL-6 levels were significantly higher than in healthy subjects. IL-8 levels decreased significantly during first five days after trauma. Nevertheless, IL- 8 values were significantly higher than in controls on the first, second, and fifth day after trauma. CRP levels in the first day after were significantly lower than on the second and fifth day. No statistical differences in CRP levels between the second and fifth day was found (Fig. 1).

No correlation between analysed cytokines and patient's sex was found.

Statistically significantly higher levels of IL-8 on the fifth day after injury were revealed in patients over 60 years of age compared to younger patients. There was no correlation with age and other studied cytokines levels. Statistically significant correlation between the value of the ISS and IL-8 on the second day after injury was observed. Signifi-
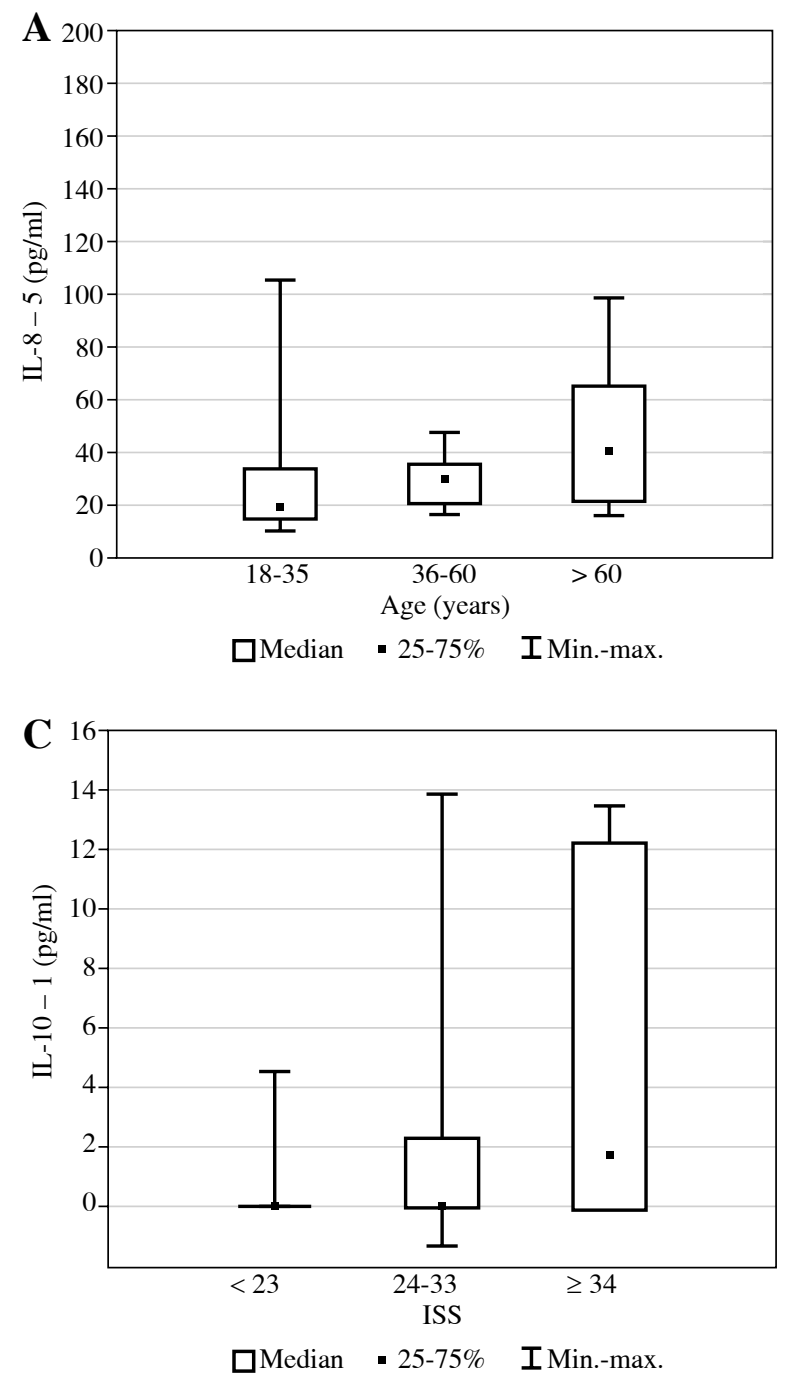

cantly higher levels of IL-6 and IL-10 on the first day after injury in patients with ISS $>34$ was observed (Fig. 2).

Significantly higher levels of IL- 8 and IL-10 on the second day after injury were revealed in patients with abdominal injuries and IL-6 in the first day after trauma in patients with limb injuries (Fig. 3). Patients with high IL-6 on the first day after trauma and limb injuries had higher incidence of infectious complications. In patients with abdominal injury the incidence of infection was higher only in cases of higher IL-8 levels on the fifth day after trauma. There was no correlation between other studied cytokines levels and the type of injury.

The presence of chronic diseases before trauma did not influence posttraumatic cytokines levels. There was no correlation of cytokine levels and blood product infusions in the early period after trauma. No association of cytokines levels and the time of first surgery was found.

A positive statistical correlation between the level of IL- 8 on the fifth day after the injury and IL- 6 on the first day after the injury with the incidence of infectious com-

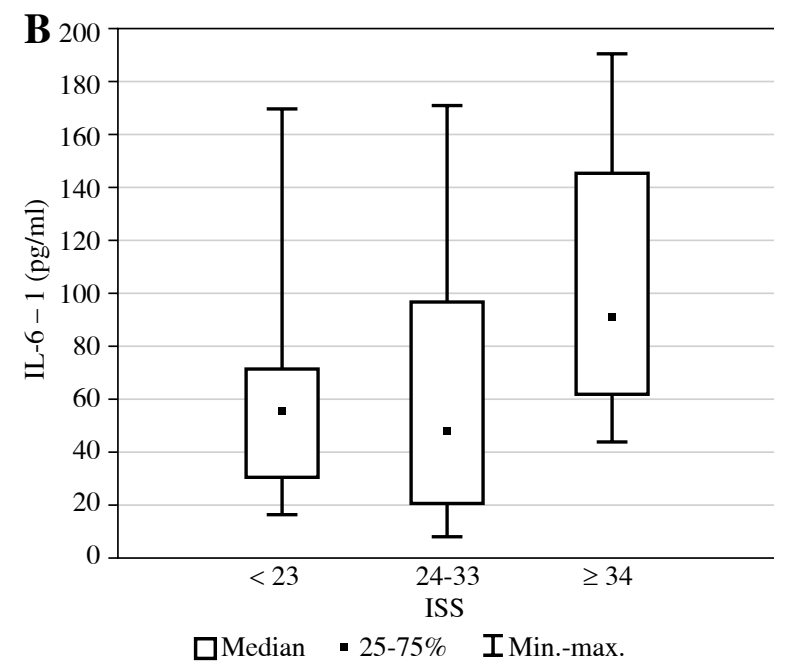

Fig. 2. A) Levels of IL-8 on the fifth day after the injury in the different age ranges. B) Levels of IL-6 on the first day after injury in different groups according to Injury Severity Score (ISS) values. C) Levels of IL-10 on the first day after injury in different groups according to ISS values 

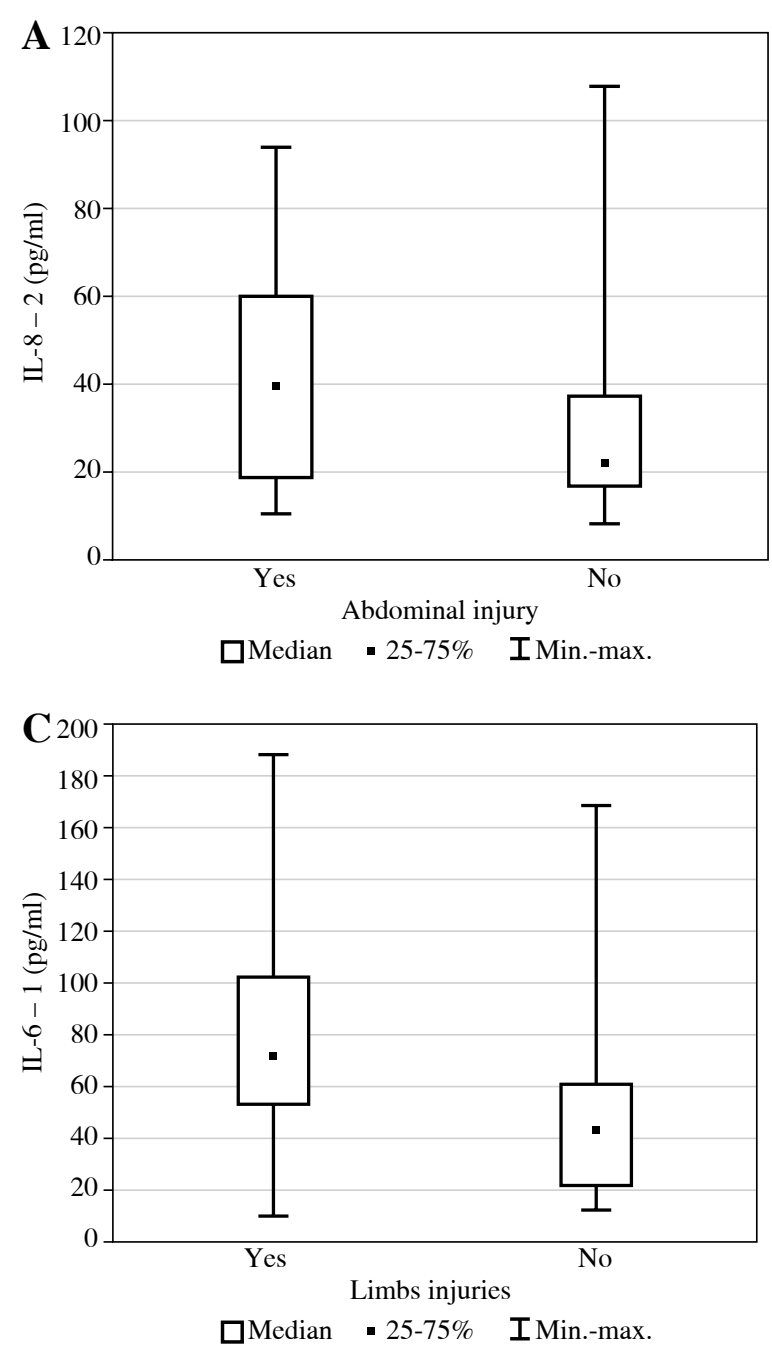

plications in patients with multiple injuries was revealed. Analysis of sensitivity and specificity showed a cut-off point of IL-6 level on the first day after injury at the level of $63.5-68.75 \%$ sensitivity and $65.2 \%$ specificity.

Statistically significantly higher values of CRP on the second day after injury predicted infectious complications in further periods of hospitalisation (wound infection, pneumonia, urinary tract infection). Analysis of sensitivity and specificity showed a cut-off point of CRP level in the second day after injury at the level of 154.4-50.0\% sensitivity and $93.5 \%$ specificity (Fig. 4 ).

\section{Discussion}

The role of cytokines in the inflammatory response is well understood. They are among the most important activators, modulators, and inhibitors of inflammation secreted by endothelial cells and leukocytes in response to tissue damage.

Severe injuries, particularly multiple, strongly stimulate the secretion of cytokines, which often leads to ex-

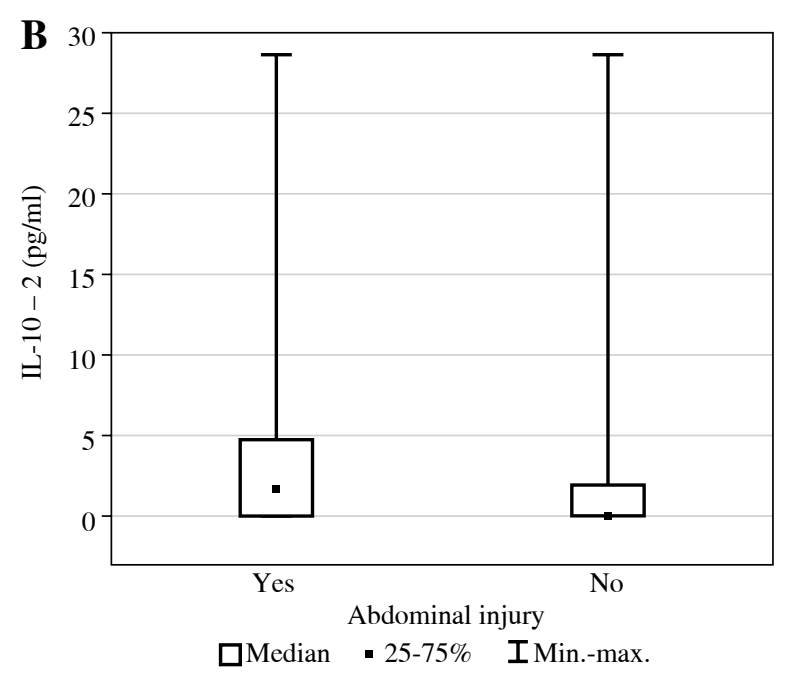

Fig. 3. Box-and-whisker plots present: A) IL-8 levels on the first day after injury, depending on the prevalence of abdominal injuries; B) IL-10 levels on the second day after injury, depending on the prevalence of abdominal injuries; C) IL-6 levels on the first day after injury, depending on the prevalence of limbs injuries

cessive intensification of inflammation, leading to the development of SIRS but also in later stage CARS (Compensatory Anti-inflammatory Response Syndrome) that can lead to multiple organ dysfunction or failure and serious infectious complications including sepsis [1, 4, 13, 14].

In the very early phase after injury TNF and IL-1 $\beta$ play a central role. However, their concentration increases as rapidly as it decreases. As a result, only in a few studies, in which samples were taken at the time of admission to the emergency department, were high levels of those substances revealed $[4,7,12,13,15]$.

In this study elevated levels of TNF and IL-1 $\beta$ were not observed even on the first day after injury, but blood samples were collected after initial diagnosis and stabilisation of patients, usually a few hours after admission.

TNF- $\alpha$ and IL- $1 \beta$ stimulate the secretion of pro-inflammatory cytokines IL-6 and IL-8. Their growth was observed in several studies concerning single and multiple injuries, and also the reaction to planned surgery. The highest levels were observed during the first 2-3 days 


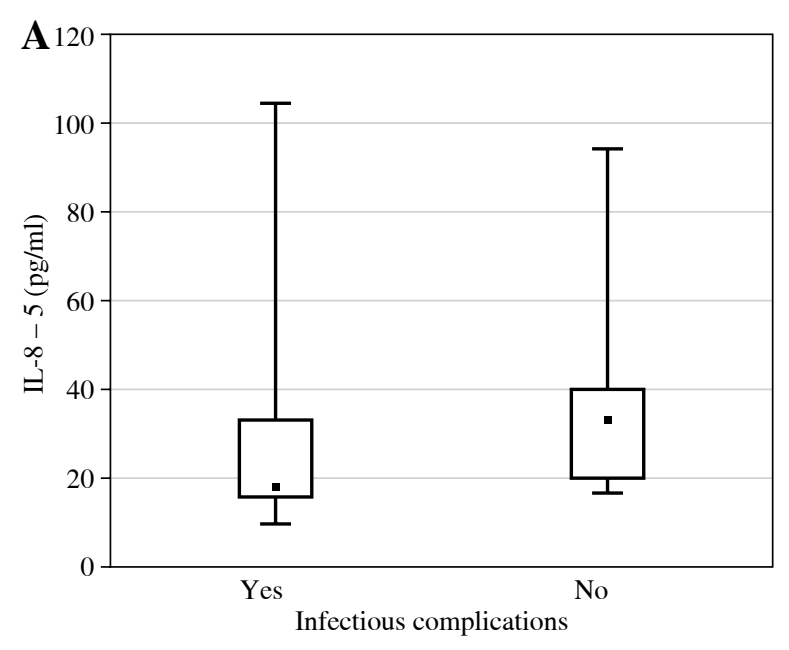

$\square$ Median $\quad$ 25-75\% IMin.-max.

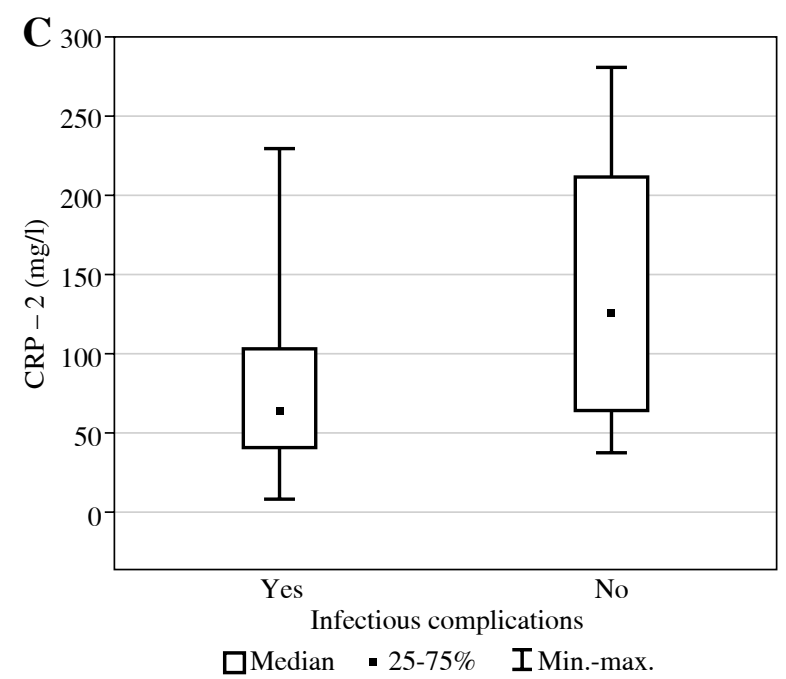

after injury with subsequent decrease on the fifth day $[4,7$, 16-18]. In our research the same course of changes in the levels of IL-6 and IL-8 was observed.

Only individual research projects have studied cytokines levels after trauma in the elderly, but without comparison to other age groups. In our study, however, higher levels of IL- 8 on the fifth day after trauma in patients over 60 years old had a positive correlation with infectious complications [19].

The dependence of IL- 6 and IL-8 levels on the severity of injuries, expressed with ISS, has been emphasised in many studies [1, 20-22]. In our research patients with ISS $>34$ had the highest levels of IL-6 on the first day after injury. In addition, in these patients the highest levels of IL-10 on the fifth day after the injury was observed, which may indicate indirectly a strong immunosuppression because it is one of the major anti-inflammatory cytokines.

Research in patients with severe trauma showed higher values of IL-6 and IL-8 in patients with multiple injuries,

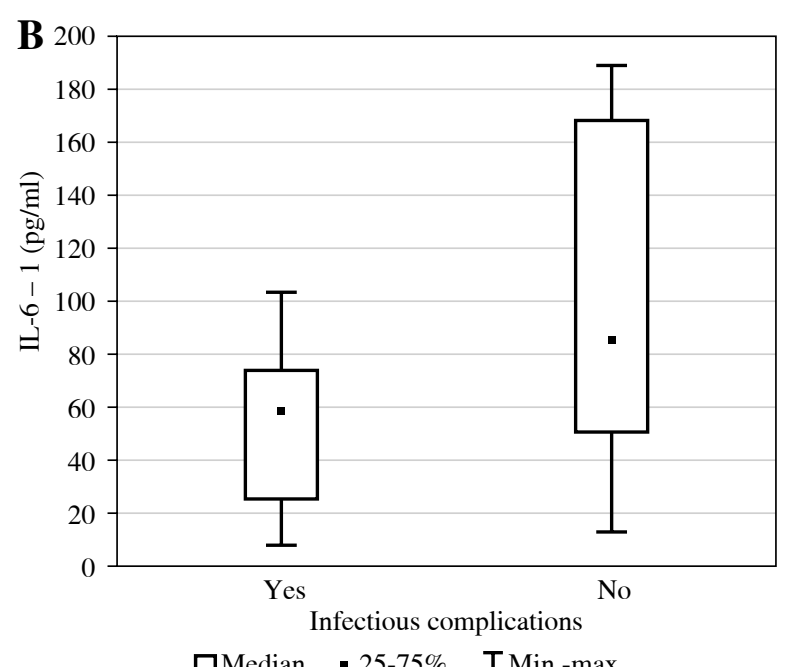

口Median : 25-75\% IMin.-max.

Fig. 4. Box-and-whisker plots present: levels of IL-8 in the fifth day after injury (A), IL-6 in the first day after injury $(\mathbf{B})$, and $\mathrm{C}$-reactive protein (CRP) in the second day after injury $(\mathbf{C})$, depending on the prevalence of infectious complications

including head and/or chest trauma, within the first 48 hours after injury [10, 23].

Such correlations did not occur in our research. Nevertheless, IL-6 levels in the first and second day after injury were higher in patients with abdominal and musculoskeletal injuries. Also, IL-10 levels on the second day were higher in patients with injuries of the abdominal cavity. The lack of correlation of head injuries with tested cytokine levels may result from the fact that these injuries occurred in $76 \%$ of patients. It is therefore difficult to achieve a statistically significant comparison.

In individual studies, higher levels of IL-6 in patients who were treated with massive blood transfusions ( $>15$ units of packed red blood cells [PRBCs]) was revealed [13]. In our study no correlation between cytokine levels and transfusions was found. However, patients included in the study did not require such excessive blood supplementation. Research concerning the effect of transfusion on the immune system (not related to trauma patients) showed an increase in anti-inflammatory 
cytokines, including IL-10 after the application of blood products [24-27]. In our study such a relation was not observed.

Despite the confirmed effect of surgical trauma on some parameters of the immune system, including cytokine levels $[19,28,29]$, in our study no influence of the time of the first surgery on the secretion of tested cytokines was revealed. Perhaps the stimulation caused by multiple trauma is so excessive that the subsequent additional surgical injury was not able to initiate a more severe reaction.

In most studies correlation between IL-6 and IL-8 levels in the early phase after injury and the prognosis for the development of multiple organ failure, survival, and the prevalence of infections, considered as one of the major causes of death in patients after trauma in the further period, has been demonstrated [14, 21, 28, 30].

In our research mortality was not analysed because, fortunately, all patients survived the effects of the injury. However, a correlation of IL-6 levels in the first day after trauma and the occurrence of infectious complications in patients with multiple injuries was revealed.

It is worth noting that IL-6 is a major activator of secretion of CRP. Our findings indicate correlation of CRP in the second day after injury with the occurrence of infectious complications. In some trauma centres, especially in Germany, monitoring of IL-6 levels in patients with multiple injuries has been introduced [1, 21, 22].

In our study correlation of IL-6 on the first day after injury and infection was demonstrated. The study showed higher sensitivity of this test as compared to CRP in the second day after injury (68.75\% for IL-6 and 50\% for CRP) but much lower specificity $-65.2 \%$ for IL-6 with $93.5 \%$ for CRP.

In our research higher levels of IL-8 on the fifth day after injury in patients who developed infectious complications was revealed. Similar results were observed in other studies $[1,6,11,13]$. In patients with multiple injuries, the increase in IL-8 levels could have been the result of already present infection in the latent phase.

Some studies also showed a correlation between IL-10 levels and complications of the respiratory system - both infectious and ARDS (Acute Respiratory Distress Syndrome) $[11,13]$. In our study, a similar dependence was not found.

\section{Conclusions}

Severe trauma resulting in multiple injuries significantly affects the course of the inflammatory response. This leads to higher levels of proinflammatory cytokines - IL-6 and IL-8 and the main anti-inflammatory cytokine IL-10.

In patients with multiple injuries the highest levels of IL-6 in first day after injury occurred in patients with ISS $>34$, and they correlated positively with the incidence of infectious complications. The values IL- 8 on the fifth day after trauma also correlated with infections and were highest in patients over 60 years of age. This can lead to the conclu- sion that older patients and those with ISS $>34$ are initially the most vulnerable to infectious complications and require a broader context of prevention activities.

The most severe dysfunction of selected parameters of the immune system in patients with multiple injuries appears in the first two days after trauma. However, even on the fifth day the immune system is still not fully efficient. Monitoring of IL-6 levels may be useful to find the optimal time for elective procedures.

Monitoring of both parameters may help to identify patients susceptible to infection in the first two days of hospitalisation with great clinical importance.

The correlation of IL- 6 and IL-8 with the occurrence of posttraumatic infectious complications was revealed. The most useful parameter is the IL-6 level on the first day after injury and the CRP level on the second day. Monitoring of both parameters may help to identify patients susceptible to infection in the first two days of hospitalisation, with great clinical importance.

The authors declare no conflict of interests.

\section{References}

1. Faist E, Wichmann MW (1997): Immunologie bei Schwerverletzten. Chirurg 68: 1066-1070.

2. Giannoudis PV (2003): Current concepts of the inflammatory response after major trauma: an update. Injury 34: 397-404.

3. van Griensven M, Krettek C, Pape HC (2003): Immune Reactions after Trauma. Eur J Trauma 29: 181-192.

4. Hranjec T, Swenson BR, Dossett LA, et al. (2010): Diagnosis-Dependent Relationships between Cytokine Levels and Survival in Patients Admitted for Surgical Critical Care. J Am Coll Surg 210: 833-844.

5. Jastrow KM, McGuire MF, Gonzalez EA, et al. (2009): Cytokine profiling: A tool for predicting early MOF in trauma patients. J Am Coll Surg 209: 320-331.

6. Mimasaka S, Funayama M, Hashiyada M, et al. (2007): Significance of levels of IL-6 and IL-8 after trauma: A study of 11 cytokines post-mortem using multiplex immunoassay. Injury 38: 1047-1051.

7. Reikerĺs O (2010): Immune depression in musculoskeletal trauma. Inflamm Res 59: 409-414.

8. Butcher N, Balogh ZJ (2009): The definition of polytrauma: the need for international consensus. Injury 40 (Suppl 4): S12-S22.

9. Morley J, Kossygan K, Giannoudis PV (2002): Damage control orthopaedics: a new concept in the management of the multiply injured patient. Current Orthopaedics 16: 362-367.

10. Tien H, Chub PTY, Brennemanc F (2004): Causes of death following multiple trauma. Current Orthopaedics 18: 304-310.

11. Seekamp A, van Griensven M, Lehmann U, et al. (2002): Serum IL-6, IL-8 and IL-10 Levels in Multiple Trauma Compared to Traumatic Brain Injury and Combined Trauma. Eur J Trauma 3: 183-189.

12. Yao Y-M, Redl H, Bahrami S, et al. (1998): The inflammatory basis of trauma/shock-associated multiple organ failure. Inflamm Res 47: 201-210. 
13. Hildebrand F, Frink M, Mommsen P, et al. (2007): Bedeutung des Immunmonitorings in der unfallchirurgischen Intensivmedizin. Trauma Berufskrankh 3: 196-200.

14. Frank J, Maier M, Koenig J, et al. (2002): Circulating Inflammatory and Metabolic Parameters to Predict Organ Failure after Multiple Trauma: Eur J Trauma 28: 333-339.

15. Cederberg D, Siesjö P (2010): What has inflammation to do with traumatic brain injury? Childs Nerv Syst 26: 221-226.

16. Walsh DS, Thavichaigarn P, Pattanapanyasat K, et al. (2005): Characterization of circulating monocytes expressing HLADR or CD71 and related soluble factors for 2 weeks after severe, non-thermal injury. J Surg Res 129: 221-230.

17. Wright EH, Khan U (2009): Serum complement-reactive protein (CRP) trends following local and free-tissue reconstructions for traumatic injuries or chronic wounds of the lower limb. J Plast Reconstr Aesthet Surg 63: 1519-1522.

18. Brunengraber LN, Robinson AV, Chwals WJ (2009): Relationship of serum C-reactive protein and blood glucose levels with injury severity and patient morbidity in a pediatric trauma population. J Pediatr Surg 44: 992-996.

19. Sedlár M, Kudrnová Z, Erhart D, et al. (2010): Older age and type of surgery predict the early inflammatory response to hip trauma mediated by interleukin-6 (IL-6). Arch Gerontol Geriatr 51: e1-6.

20. Perl M, Huber-Lang M, Gebhard F (2012): The influence of coagulation and inflammation research on the improvement of polytrauma care. Eur J Trauma Emerg Surg 38: 231-239.

21. Bauer C, Ketter R, Silomon M (2008): Einfluss moderner Laborparameter auf das klinische Management des polytraumatisierten Patienten. Intensivmed 2: 64-69.

22. Hildebrand F, Frink M, Mommsen P, et al. (2007): Bedeutung des Immunmonitorings in der unfallchirurgischen Intensivmedizin. Trauma Berufskrankh 9: 196-200.

23. Maier M, Geiger EV, Wutzel S, et al. (2009): Role of Lung Contusions on Posttraumatic Inflammatory Response and Organ Dysfunction in Traumatized Patients. Eur J Trauma Emerg Surg 35: 463-469.

24. Brand A (2002): Immunological aspects of blood transfusions. Transpl Immunol 10: 183-190.

25. Matinlauri I (2004): Immunological aspects of blood transfusion preparations. Duodecim 120: 867-875.

26. Flohé S, Kobbe P, Nast-Kolb D (2007): Immunological reactions secondary to blood transfusion. Injury 38: 1405-1408.

27. Lannan KL, Sahler J, Spinelli SL, et al. (2013): Transfusion immunomodulation - the case for leukoreduced and (perhaps) washed transfusions. Blood Cells Mol Dis 50: 61-68.

28. Sido B, Teklote JR, Hartel M, et al. (2004): Inflammatory response after abdominal surgery. Best Pract Res Clin Anaesthesiol 18: 439-454.

29. Dunker MS, Ten Hove T, Bemelman WA, et al. (2003): Interleukin-6, C-reactive protein, and expression of human leukocyte antigen-DR on peripheral blood mononuclear cells in patients after laparoscopic vs. conventional bowel resection: a randomized study. Dis Colon Rectum 46: 1238-1244.

30. Maier B, Lefering R, Lehnert M, et al. (2007): Early versus late onset of multiple organ failure is associated with differing patterns of plasma cytokine biomarker expression and outcome after severe trauma. Shock 28: 668-674. 